\title{
Factor V Leiden and the Risk of Pulmonary Embolism
}

\author{
Shrestha Mukesh, Li Wei*
}

Department of Pulmonary and Critical Care, The First Affiliated Hospital of Kunming Medical University, Kunming, Yunnan, P.R China.

*Corresponding Author: Li Wei, Department of Pulmonary and Critical Care, The First Affiliated Hospital of Kunming Medical University, Kunming, Yunnan, 650032, P.R China

Received: 31 December 2018; Accepted: 10 January 2019; Published: 14 January 2019

\begin{abstract}
Venous thromboembolism (VTE) is the third most common cardiovascular disease which is an under-appreciated contributor to the global burden of non-communicable diseases. Previously pulmonary embolism (PE) and deep vein thrombosis (DVT) were regarded as a single disease entity and still today, public awareness of DVT and PE remains comparatively low. But after recognition of genetic factors, such as Factor V Leiden (FVL), the concept has been changing. More studies are going on to observe the correlation between the genetic factors, such as FVL and PE. Although there is some biasness regarding the concept of factors associated with the PE, the role of possible genetic factors (FVL) remains the main priority in our review.
\end{abstract}

Keywords: Pulmonary embolism; Deep vein thrombosis; Venous thromboembolism; Factor V Leiden (FVL); PT G20210A

Abbreviations: APC: activated protein C; Arg 506: amino acid arginine at position 506 in factor V; DVT: deep vein thrombosis; FVL: Factor V Leiden; PE: pulmonary embolism; PT G20210A: prothrombin G20210A; RV: right ventricular; VTE: venous thromboembolism

\section{Introduction}

Pulmonary embolism (PE) is an obstructive disease of the pulmonary arterial system commonly occurring due to the embolization of thrombus originating from the deep veins of the lower extremities [1, 2]. It is one of the leading causes of mortality globally [3]. The mortality rate is $30-35 \%$ if acute PE is left untreated [4], $8 \%$ for treated patient and 2$50 \%$ have been reported for the recurrence after treating correctly [5]. Since, life-threatening acute PE is an outcome of 
hemodynamic instability due to the combination of size of the emboli and the underlying cardiopulmonary status [6], it is vitally important for clinicians to make a correct clinical diagnosis of acute PE and understand the underlying pathophysiology [7]. The pathophysiology of PE comprises of direct physical obstruction of the pulmonary arteries, hypoxemic vasoconstriction, and release of potent pulmonary arterial vasoconstrictors, which contribute to rising in pulmonary vascular resistance and right ventricular (RV) afterload. Then the acute RV pressure overload could lead to RV hypokinesis and dilation, tricuspid regurgitation, and finally, RV failure [7]. Lately, European Society of Cardiology has published an update of their guideline on the diagnosis and management of acute PE [8]. According to the study conducted by Law et al. [9], the annual incidences of PE, PE with DVT, and DVT in China were 8.7 per 100,000 population, 3.0 per 100,000 population, and 30.0 per 100,000 population, respectively. The 30 -day mortality rates accompanying PE, DVT, and PE with DVT were 17.4\%, 9.0\%, and 13.3\% respectively. In the Caucasian population, the annual incidence rate of PE is between 45-189 per 100,000 population. Whereas, the annual incidence rates among Asians is between 3.9-7 per 100,000 population for PE [10,11]. Currently, VTE is considered to be one of the major, but underappreciated factors leading to the preventable hospital deaths globally [3] and the incidence rate has increased over time in Asia [12]. Hence, there is a substantial necessity for further research and review in Asian population regarding DVT and PE. The various factors, such as environmental, clinical and genetic, play a significant role for the pathogenesis of VTE/PE. Due to this reason, genetics was not given much attention earlier, however, the concept changed when FV Leiden was recently described as an abnormality of coagulation and found to be associated with $18-20 \%$ of all idiopathic VTE cases $[13,14,15]$.

\section{What is a Factor V Leiden?}

Human coagulation factor $\mathrm{V}(\mathrm{FV})$ is a crucial coagulation protein and plays an essential role in the procoagulant and anticoagulant pathways [16]. Whereas, activated protein C (APC), with its cofactor protein S, inactivates factors Va and VIIIa to provide a major natural anticoagulant system [17]. Dählback et al. [18] first described the APC resistance caused by FVL in 1993 and currently, this is the most commonly acknowledged hereditary predisposition to venous thrombosis [17, 18]. FVL refers to the specific guanine to adenine substitution at nucleotide 1691 in the Factor V gene, which predicts the substitution of glutamine for arginine at the Arg 506 APC cleavage site. As a result, Factor Va is resistant to APC and is inactivated at a 10-fold slower rate than normal, leading to increased thrombin generation. FVL is a genetic disorder characterized by an inadequate anticoagulant response to activated protein $\mathrm{C}$ and increases the risk of DVT and PE (20 -25\% of patients with VTE). The current studies suggest that the mutation has a fundamental role in recurrence risk after initial treatment of first VTE. But the DNA analysis of the F5 gene that encodes the Factor V protein or the protein $C$ resistance assay is required for the diagnosis [19]. In 90-95\% of cases, the phenotypic diagnosis can be directly correlated with FV Leiden [20]. Several analyses have shown that the prevalence of the mutation differs depending on races, ranging from 5.27-11\% in European descent to approximately $1 \%$ in those of African descent [20-22]. According to the data derived from one case-control study of African-Americans in Georgia, a FVL prevalence rate was almost comparable in both cases and controls (1.2\%) [22]. Hence, the association between FVL and VTE profoundly depend on ethnicity. 


\section{Role of FVL in PE}

Even though VTE has a wide range of clinical manifestations and a multiple risk factors, DVT and its major complication PE, are generally considered as a single disease entity [23]. Patients presenting with VTE were previously assumed that the hereditary and the acquired risk factors in patients presenting with DVT are similar to those in patients presenting with PE [24]. So to validate this assumption, Manten et al. [24] conducted a study comparing the correlation of FVL with the different clinical presentations of venous thromboembolic disease. The result showed that the prevalence of the FVL mutation was lowest in patients with Symptoms of PE only and highest in patients with DVT only, whereas the prevalence was in between in patients with Symptoms of both. In a patient without acquired risk factors, FVL was present in $22.7 \%$ of the patients presenting with DVT only, and in $11.5 \%$ of the patients with PE only. However, with acquired risk factors, $33.3 \%$ of the patients presented with PE only and $18.5 \%$ of the patients had DVT only. In patients with clinical features of both, this percentage was 13. Overall, the FVL increased the risk of PE by three-fold, and DVT by seven-fold in the carriers of FVL. Similarly, another study conducted by Turkstra et al. [25] reported that the prevalence of the FVL mutation was half of that reported in patients with primary DVT. The common coagulation disorder predisposing to venous thrombosis (resistance to activated protein) was not found to be related with an increased risk for PE and no additional information about concomitant DVT was provided as the evidence was totally based on a functional assay to diagnose APC resistance. So Martinelli et al. [26] carried out a study for the evaluation of the prevalence of factor V by finding out the gene mutation responsible for APC resistance. In that study, the prevalence of FVL was found to be $4.9 \%$ (41 patients) with PE only, while $16.9 \%$ (65 patients) in patients with PE with DVT. Similarly, Hirmerova et al. [27] found that FVL positive had a substantially higher prevalence of isolated symptomatic DVT in comparison to symptomatic PE with or without DVT. However, the increasing age and the unprovoked nature of the VTE event carried a greater risk of symptomatic PE. On the contrary, the result of the study conducted by the Ridker and colleagues [15] showed that the increased risk of prevalence of FVL mutation appeared in the primary venous thrombosis (DVT, PE or both) rather than secondary venous thrombosis and the effects were most apparent among older men. Likewise, until now most research on the risk of the mutation has been done on patients with deep venous thrombosis of the legs or non-fatal pulmonary emboli [28]. So there has been an important question whether or not carriers have an equally elevated risk of developing fatal pulmonary emboli in the presence as well as in the absence of acquired risk factors. As many data regarding the interaction between the factor $\mathrm{V}$ mutation and acquired risk factors for thrombosis has shown different effects. So, Vandenbroucke et al. [28] carried out a study using pathology material from the autopsies of patients to investigate whether the FVL mutation aggravates the risk of fatal pulmonary emboli. The first series of the study consisted of consecutive autopsies in which pulmonary emboli were mentioned in the autopsy report (most patients of this series had the major underlying disease). The second series consisted of autopsies in patients below 70 years of age in which pulmonary emboli were the only cause of death and no additional major acquired risk factor for venous thrombosis was present. The study showed that in the first series, the presence of FVL was determined in 44 patients, 1 of whom carried the mutation (2.3\%). This prevalence was not different from the general population of that place. In the second series, FVL was detected in 30 patients of whom 3 carried the mutation (10\%) that lead to a threefold relative risk. So, the study concluded that in the presence of severe illnesses, the FVL mutation plays no additional role in the development of pulmonary emboli. The relative risk of the 
very rare fatal pulmonary embolus, which is the only cause of death, may also be not more than the relative risk for DVT in the carriers of the FVL mutation. Similarly, other studies regarding the relationship between FVL and PE using autopsy material found no association between them $[29,30]$.

\section{FVL and the PT G20210A variant}

A single point mutation in factor $\mathrm{V}$ gene (at nucleotide position 1,691, G-A substitution) [31] and in prothrombin gene (a G to A transition at position 20210 in the sequence of the 3-UT region) [32] were identified by Bertina et al. and Poort et al. having a relationship in VTE. These two factors are the most common associated genetic risk factors for VTE [31,32], though many studies suggest that VTE recurrence rates are higher in patients with inherited genetic defects [33,34]. A study carried out by Gadelha et al. [35] enrolling patients with the first episode of VTE showed that out of 22428, 345 patients had FVL, 261 had prothrombin G20210A, and 2399 tested negative. Similarly, according to a large meta-analysis conducted in 2012, the results suggest that FV Leiden patients are more likely to present with DVT than with isolated PE [23]. On the other hand, the prevalence of prothrombin mutation is similar in patients presenting with DVT and in patients presenting with isolated PE [23,36]. Prothrombin is less likely to affect the VTE location at presentation [23]. Hence, the reason why the prevalence of prothrombin was similar in patients presenting with DVT and in patients presenting with isolated PE remains to be established. Another study which was conducted in China reported that either FVL or prothrombin G20210A mutation was not determined in their study. The result that they obtained was very similar with those from other Asian countries Japan, Korea, and Thailand, and suggested that FVL and prothrombin G20210A mutations are very rare in Asians and not genetic risk factors for both PE and DVT [37]. Furthermore, Sundquist et al. [38] conducted a study to see the relationship between VTE recurrence in relation to FVL and prothrombin mutations. In that study, $47 \%$ had FVL, compared to $31 \%$ of patients without recurrence. Prothrombin was the second most common thrombophilic defect after FVL and was present in $6 \%$ of the patients with recurrence compared to $4 \%$ in patients without recurrence.

\section{The Relationship between PE and DVT}

Numerous risk factors are known to be associated with the venous thrombosis. Advanced age, surgery, fractures, prolonged immobilization, use of oral contraceptives and hormone replacement therapy, pregnancy, puerperium, cancer, and antiphospholipid syndrome are the generally accepted acquired risk factors [37]. The relative risk of PE is found to be lower than that of DVT in patients with APC resistance and the prevalence of FVL or APC resistance in patients with isolated PE has been reported to be about half of that in patients with isolated DVT $[24,25,26]$. Whereas, DVT and PE patients do not differ in the prevalence of the second most common thrombogenic mutation, the G20210A prothrombin mutation [23,36]. According to Bounameaux [36], there are two main hypotheses for the FVL for DVT and PE. Firstly, the lower prevalence of the APC resistance had been found in unselected PE patients. This might be because of the selection bias as most of the data on FVL and DVT were coming from highly specialized centers. Secondly, the thrombus is more stable and more adherent to the vessel wall in patients with FVL and this might be perhaps due to the fact that the factor augments the local thrombin generation which further intensifies the local inflammatory process to the thrombus, or might be because it impairs the profibrinolytic response to APC. This 
second hypothesis is further supported by the Björgell and colleagues [39]. They have described and compared the location and extent of thrombosis of in FVL carriers and non-carriers with DVT and found that thrombosis occurred less often in the iliofemoral veins among FVL carriers and also the thrombi in those patients were significantly smaller than in the non-carriers. As PE originates more frequently from large, proximal (iliofemoral) thrombi, the patients with FVL might be less prone than those without to develop PE when they have DVT. Similarly, Goldhaber [40] has mentioned the upper extremity DVT as a frequently overlooked problem as it has become more common with the increasing use of central venous catheters, pacemakers, and internal cardiac defibrillators. The study highlights the risk of DVT varying with the site of catheter insertion and regardless of catheter location, all thromboses related to central venous catheter placement have the potential to embolize and cause PE. Similarly another study in 1999 conducted on patients from 7 different countries (United States, Mexico, Italy, France, Switzerland, Poland, Belgium) and concluded that PE remains an important clinical problem with a high mortality rate and thus identification of prognostic indicators for poor outcomes in those patients benefits them from receiving more aggressive interventions, such as thrombolysis or embolectomy [41].

\section{Conclusion}

The prevalence of FVL is more in DVT in comparison to the PE alone, and the opposite is true for the patient with the acquired risk factors. In the presence of severe illnesses, the FVL plays no additional role in the development of pulmonary emboli and the relative risk of the very rare fatal pulmonary embolus may possibly be less than the deepvein thrombosis in carriers of the FVL mutation. While the prevalence of prothrombin mutation is the second most common thrombophilic defect after FVL in patients with recurrent VTE, there is no difference in prothrombin mutation in DVT and isolated PE patients. Although many studies have been performed to establish the relationship between FVL and PE, there is a need of the large prospective studies to establish the associations between them due to the reason of avoiding biasness in the selection of the true patients and related risk factors.

\section{References}

1. Dalen JE. Pulmonary embolism: what have we learned since Virchow? Natural history, pathophysiology and diagnosis. Chest 122 (2002): 1440-1456.

2. Kearon C. Natural history of venous thromboembolism. Circulation 107 (2003): I22-I30.

3. The Lancet Haematology 2 (2015): e393.

4. Dalen JE. Pulmonary Embolism: What Have We Learned Since Virchow?* Treatment and Prevention. CHEST 122 (2002): 1801-1817.

5. Carson JL, Kelley MA, Duff A, et al. The clinical course of pulmonary embolism. N Engl J Med 326 (1992): 1240-1245.

6. Wood KE. Major pulmonary embolism: review of a pathophysiologic approach to the golden hour of hemodynamically significant pulmonary embolism. Chest 121 (2002): 877-905.

7. Pelliccia F, Schiariti M, Terzano C, et al. Treatment of acute pulmonary embolism: update on newer pharmacologic and interventional strategies. Biomed Res Int 2014: 410341. 
8. Konstantinides SV, Torbicki A, Agnelli G, et al. 2014 ESC Guidelines on the diagnosis and management of acute pulmonary embolism: the task force for the diagnosis and management of acute pulmonary embolism of the European Society of Cardiology (ESC). Eur Heart J 35 (2014): 3033-3069.

9. Law Y, Chan YC, Cheng SWK. Epidemiological updates of venous thromboembolism in a Chinese population. Asian J Surg 41 (2018): 176-182.

10. Cheuk BL, Cheung GC, Cheng SW. Epidemiology of venous thromboembolism in a Chinese population. Br J Surg 91 (2004): 424-428.

11. Jang MJ, Bang SM, Oh D. Incidence of venous thromboembolism in Korea: from the Health Insurance Review and Assessment Service database. J Thromb Haemost 9 (2011): 85-91.

12. Lee LH, Gallus A, Jindal R, et al. Incidence of Venous Thromboembolism in Asian Populations: A Systematic Review. Thromb Haemost 117 (2017): 2243-2260.

13. De Stefano V, Chiusolo P, Paciaroni K, Leone G. Epidemiology of factor V Leiden: clinical implications. Seminar Thromb Hemost 24 (1998): 367-379.

14. Price DT, Ridker PM. Factor V Leiden mutation and the risks for thromboembolic disease: A clinical perspective. Ann Intern Med. 127 (1997): 895-903.

15. Ridker PM, Hennekens Ch H, Lindpaintner K, et al. Mutation in the gene coding for coagulation factor V and the risk of myocardial infarction, stroke, and venous thrombosis in apparently healthy men. N Engl J Med 332 (1995): 912-917.

16. Segers K, Dahlbäck B, Nicolaes GA. Coagulation factor V and thrombophilia: background and mechanisms. Thromb Haemost 98 (2007): 530-542.

17. Van Cott EM, Khor B, Zehnder JL. Factor V Leiden. Am J Hematol 91 (2016): 46-49.

18. Dahlback B, Carlsson M, Svensson PJ. Familial thrombophilia due to a previously unrecognized mechanism characterized by poor anticoagulant response to activated protein C: Prediction of a cofactor to activated protein C. Proc Natl Acad Sci USA 90 (1993): 1004-1008.

19. Kujovich JL. Factor V Leiden thrombophilia. Genet Med 13 (2011): 1-16.

20. Hooper WC, Evatt BL. The role of activated protein C resistance in the pathogenesis of venous thrombosis. Am J Med Sci 316 (1998): 120-128.

21. Ridker PM, Miletich JP, Hennekens CH, et al. Ethnic distribution of Factor V leiden in 4047 men and women. Implicationsnfor venous thrombosis screening. JAMA 277 (1997): 1305-1307.

22. Hooper WC, Dilley A, Ribeiro MJA, et al. Racial difference in the prevalence of the Arg506 $\rightarrow$ Gln mutation. Thromb Res 81 (1996): 577-581.

23. Dentali F, Ageno W, Bozzato S, et al. Role of factor V Leiden or G20210A prothrombin mutation in patients with symptomatic pulmonary embolism and deep vein thrombosis: a meta-analysis of the literature. J Thromb Haemost 10 (2012): 732-737.

24. Manten B, Westendorp RG, Koster T, et al. Risk factor profiles in patients with different clinical manifestations of venous thromboembolism: a focus on the factor V Leiden mutation. Thromb Haemost 76 (1996): 510-513. 
25. Turkstra F, Karemaker R, Kuijer PM, et al. Is the prevalence of the factor V Leiden mutation in patients with pulmonary embolism and deep vein thrombosis really different?. Thromb Haemost 81 (1999): 345-348.

26. Martinelli I, Cattaneo M, Panzeri D, et al. Low prevalence of factor V: Q506 in 41 patients with isolated pulmonary embolism. Thromb Haemost 77 (1997): 440-443.

27. Hirmerova J, Seidlerova J, Subrt I. The association of factor V Leiden with various clinical patterns of venous thromboembolism-the factor V Leiden paradox. QJM. 107 (2014): 715-720.

28. Vandenbroucke JP, Bertina RM, Holmes ZR, et al. Factor V Leiden and fatal pulmonary embolism. Thromb Haemost 79 (1998): 511-516.

29. Gorman TE, Arcot A, Baker P, et al. Prevalence of the factor V Leiden mutation among autopsy patients with pulmonary thromboembolic disease using an improved method for Factor V Leiden detection. Am J Clin Pathol 11 (1999): 413-417.

30. Slovacek KJ, Harris AF, Greene JF, et al. Fatal pulmonary embolism: A study of genetic and acquired factors. Molec Diag 5 (2000): 53-58.

31. Bertina RM, Koeleman BP, Koster T, et al. Mutation in blood coagulation factor V associated with resistance to activated protein C. Nature 369 (1994): 64-67.

32. Poort SR, Rosendaal FR, Reitsma PH, et al. A common genetic variation in the $3 \phi$-untranslated region of the prothrombin gene is associated with elevated plasma pro-thrombin levels and an increase in venous thrombosis. Blood 88 (1996): 3698-3703.

33. De Stefano V, Simioni P, Rossi E, et al. The risk of recurrent venous thromboembolism in patients with inherited deficiency of natural anticoagulants antithrombin, protein C and protein S. Haematologica 91 (2006): 695-698.

34. Sveinsdottir SV, Saemundsson Y, Isma N, et al. Evaluation of recurrent venous thromboembolism in patients with Factor V Leiden mutation in heterozygous form. Thromb Res 130 (2012): 467-471.

35. Gadelha T, Roldán V, Lecumberri $\mathrm{R}$, et al. Clinical characteristics of patients with factor $\mathrm{V}$ Leiden or prothrombin G20210A and a first episode of venous thromboembolism. Thrombosis Research 126 (2010): 283286.

36. Bounameaux H. Factor V Leiden paradox: risk of deep vein thrombosis but not of pulmonary embolism. Lancet 356 (2000): 182-183.

37. Jun ZJ, Ping T, Lei Y, et al. Prevalence of factor V Leiden and prothrombin G20210A mutations in Chinese patients with deep venous thrombosis and pulmonary embolism. Clin Lab Haematol 28 (2006): 111-116.

38. Sundquist K, Wang X, Svensson PJ, et al. Plasminogen activator inhibitor-1 4G/5G polymorphism, factor V Leiden, prothrombin mutations and the risk of VTE recurrence. Thromb Haemost 114 (2015): 1156-1164.

39. Björgell O, Nilsson PE, Nilsson JA, et al. Location and extent of deep vein thrombosis in patients with and without FV:R506Q mutation. Thromb Haemost 83 (2000): 648-651.

40. Goldhaber SZ. DVT Prevention: What Is Happening in the "Real World"? Semin Thromb Hemost 29 (2003): 023-032. 
41. Goldhaber SZ, Visani L, De Rosa M. Acute pulmonary embolism: clinical outcomes in the International Cooperative Pulmonary Embolism Registry (ICOPER). Lancet 353 (1999): 1386-1389.

Citation: Shrestha Mukesh, Li Wei. Factor V Leiden and the Risk of Pulmonary Embolism. Cardiology and Cardiovascular Medicine 3 (2019): 001-008. 